\title{
Review of: "TNF- $\alpha$-mediated m6A modification of ELMO1 triggers directional migration of mesenchymal stem cell in ankylosing spondylitis"
}

YUKI NANKE ${ }^{1}$

1 Tokyo Women's Medical University

Potential competing interests: The author(s) declared that no potential competing interests exist.

The authors have demonstrated that TNF-alpha-mediated m6A modification of ELMO1 triggers directional migration of mesenchymal stem cell in ankylosing spondylitis (AS) using samples from AS patients and SKG mice.

Their manuscript has been well written. In addition, the findings are very interesting. Especially, the third paragraph of Discussion is interesting and excellent; 'the local inflammatory positons with higher concentration of TNF-alpha' is discussed in this paragraph. The local concentration of a cytokine is very important hypothesis in the cell biology.

I have no major concerns; however, I have several minor concerns.

1. The data about anti-IL17 and anti-IL23 neutralizing antibody should be described in the Figures of manuscript but not in Supplementary Figures, because these data are very important in the discussion of the treatment.

2. SKG mice were used as an AS model; however, SKG mice are usually a rheumatoid arthritis model for rheumatologists. Thus, the author should explain SKG mice as an AS model in more detail in this paper.

3. Page 4 , the right column, the $1^{\text {st }}$ paragraph. 'shRNA' should be mentioned in this paragraph.

4. Page 11 , the left column, the $1^{\text {st }}$ paragraph. 'a vicious three-step cycle' is very interesting hypothesis. The Authors should show a schema to explain the hypothesis in the manuscript.

5. Page 11, the last paragraph, line 4. 'the diagnosis' is first mentioned in the last paragraph of Discussion summarizing the manuscript. 'The diagnosis' should be discussed in the other paragraph of Discussion. 